\title{
Análise psicométrica das Escalas Nordoff-Robbins como instrumento de avaliação no tratamento musicoterapêutico de crianças autistas em acompanhamento no Hospital das Clínicas da Universidade Federal de Minas Gerais (HC-UFMG)
}

\section{Psychometric analysis of the Nordoff-Robbins Scales as an instrument of evaluation in the Music Therapy treatment of autistic children in follow up in the Hospital das Clínicas da Universidade Federal de Minas Gerais (HC-UFMG)}

\author{
Aline Moreira André \\ Universidade Federal de Minas Gerais, Belo Horizonte, Minas Gerais, Brasil \\ aline.musicasax@gmail.com
}

\section{Davi Oliveira Batista}

Universidade Federal de Minas Gerais, Belo Horizonte, Minas Gerais, Brasil davi-ba@hotmail.com

\author{
Marina Horta Freire \\ Universidade Federal de Minas Gerais, Belo Horizonte, Minas Gerais, Brasil \\ marinahf@gmail.com
}

\section{Renato Tocantins Sampaio}

Universidade Federal de Minas Gerais, Belo Horizonte, Minas Gerais, Brasil rsampaiomt@gmail.com

\section{Arthur Melo e Kummer}

Eli Lilly and Company, Belo Horizonte, Minas Gerais, Brasil

r2kummer@hotmail.com

\begin{abstract}
Resumo: 0 tratamento musicoterapêutico, ascendente forma de tratamento para o Transtorno do Espectro do Autismo, vem demonstrando grande eficácia para pacientes com autismo ou características autísticas. Os objetivos terapêuticos incluem a melhora na comunicação, na fala, na socialização e as mudanças positivas de comportamento. Entre os recursos disponíveis para avaliar a melhora clínica, existem duas escalas desenvolvidas na abordagem Nordoff Robbins (NR) que avaliam a Comunicabilidade Musical e a Relação entre terapeuta e cliente. Por intermédio destas escalas, foi realizada análise cega dos vídeos da primeira e da última sessão das crianças autistas que receberam Musicoterapia no HC-UFMG, para verificar a confiabilidade interexaminadores. Essas escalas também foram comparadas com outras ferramentas de avaliação: Childhood Autism Rating Scale (CARS), Autism Treatment Evaluation Checklist (ATEC) e Improvisational Assessment Profiles (IAPs), a fim de verificar a validade concorrente. Observou-se boa confiabilidade interexaminadores das Escalas NR e melhora na comunicabilidade musical vocal e de movimento corporal no grupo intervenção. Observou-se, ainda, correlação moderada entre as Escalas NR, CARS e ATEC e correlações fortes entre Escalas NR e IAPs.
\end{abstract}

Palavras-chave: musicoterapia e autismo; avaliação de comunicação e socialização. 
ANDRÉ, Aline Moreira; BATISTA, Davi Oliveira; FREIRE, Marina Horta; SAMPAIO, Renato Tocantins; KUMMER, Arthur Melo e (2018). Análise psicométrica das Escalas Nordoff-Robbins como instrumento de avaliação no tratamento musicoterapêutico de crianças autistas em acompanhamento no Hospital das Clínicas da UFMG (HC-UFMG). Per Musi. Belo Horizonte: UFMG. p.1-12.

\begin{abstract}
Music Therapy treatment, ascending form of treatment for Autism Spectrum Disorder, has been demonstrating efficacy for patients with autism or autistic characteristics. The therapeutic goals include improvement in communication and speech, and positive changes in behavior. Among the available resources to measure the clinical outcomes, there are two scales developed in the Nordoff Robbins approach (NR) that evaluate Musical Communicability and Client-Therapist Relationship. By using these scales, a blind analysis of video recordings from the first and last session of children with autism that received Music Therapy at HC-UFMG was conducted to verify inter-examiner reliability. These scales were also compared with other instrument of assessment: Childhood Autism Rating Scale (CARS), Autism Treatment Evaluation Checklist (ATEC) and Improvisational Assessment Profiles (IAPs) to verify concurrent validity. A good inter-examiner reliability was found along with improvements in vocal musical communicability and body movement communicability in the intervention group. There were also observed a moderate correlation between the NR scales, CARS, and ATEC; and a strong correlation between NR scales and IAPs.
\end{abstract}

Keywords: music therapy and autism; evaluation of communication and socialization.

Submission date: 28 February 2018

Final approval date: 23 June 2018

\title{
1 - Introdução
}

O Transtorno do Espectro do Autismo (TEA) é um transtorno do desenvolvimento das habilidades sociocomunicativas associado a padrão restrito e estereotipado do comportamentos e interesses (APA, 2014). Sua prevalência atual é estimada em 1 criança afetada a cada 68, sendo mais frequente naquelas do sexo masculino (BAIO et al., 2018). Quanto à etiologia, atribui-se um papel importante à genética, havendo grande possibilidade de mais de um diagnóstico na família ou de características autísticas amplamente presentes, mesmo que os indivíduos não se encaixem nos critérios diagnósticos.

Existem várias terapias que buscam auxiliar no tratamento de pacientes com autismo. Dentre elas, a Musicoterapia tem sido recomendada como um tratamento eficaz para facilitar a comunicação entre paciente e terapeuta. Segundo a Federação Mundial de Musicoterapia:

“...A musicoterapia é a utilização profissional da música e seus
elementos como uma intervenção em ambientes médicos,
educacionais e cotidianos com indivíduos, grupos, famílias ou
comunidades que buscam otimizar sua qualidade de vida e
melhorar o seu bem-estar físico, social, comunicativo, emocional,
intelectual, espiritual e de saúde...” (WFMT, 2011).

A música é um meio que envolve uma enorme variedade de expressões comunicativas e pode auxiliar para que outras formas de comunicação possam ser estabelecidas para promover interação, envolvimento e relacionamento (WIGRAM, 2006). Crianças com TEA demonstram alterações no fazer musical, assim como demonstram na sua vida diária (WIGRAM e GOLD, 2006).

A Musicoterapia tem um papel relevante no diagnóstico do autismo por meio da diferenciação de comportamentos característicos e na avaliação clínica, avaliando os pontos fortes e fracos em diversas áreas do desenvolvimento (MøLLER; ODELL-MILLER e WIGRAM, 2002). Dessa 
ANDRÉ, Aline Moreira; BATISTA, Davi Oliveira; FREIRE, Marina Horta; SAMPAIO, Renato Tocantins; KUMMER, Arthur Melo e (2018). Análise psicométrica das Escalas Nordoff-Robbins como instrumento de avaliação no tratamento musicoterapêutico de crianças autistas em acompanhamento no Hospital das Clínicas da UFMG (HC-UFMG). Per Musi. Belo Horizonte: UFMG. p.1-12.

forma, podemos observar que, além da eficácia no tratamento/reabilitação do indivíduo autista, a Musicoterapia pode exercer um relevante papel num momento anterior, o diagnóstico.

Existem várias técnicas e métodos de Musicoterapia que auxiliam no desenvolvimento de habilidades de comunicação, socialização e comportamento, que geralmente estão afetados precocemente no autismo. Estudos sugerem que o tratamento com Musicoterapia para crianças autistas pode auxiliar na qualidade da comunicação e da interação no âmbito social, terapêutico e familiar (BIELENINIK et al., 2017; FREIRE, 2014; SAMPAIO, 2015; THOMPSON; MCFERRAN e GOLD, 2014).

O avanço da Musicoterapia traz consigo a necessidade de estudos científicos sobre o seu uso na clínica do TEA, incluindo (mas não limitado a) a avaliação de sua eficácia, quais técnicas musicoterapêuticas ou componentes da intervenção são mais eficazes, preditores de resposta etc. Para isso, são necessários instrumentos específicos de Musicoterapia para confirmar a evolução clínica cientificamente. Porém, são escassos instrumentos desse tipo no Brasil.

A tradução e validação da escala KAMUTHE para o português brasileiro ofereceu evidências para que este instrumento possa ser utilizado no Brasil (GATTINO, 2012). Apesar disso, ainda existe a necessidade de mais ferramentas de mensuração validadas para a avaliação dos indivíduos com autismo no nosso país e a verificação das propriedades psicométricas das escalas que existem fora do país ainda se mostra bastante importante.

A literatura afirma que em 1966 foram desenvolvidos testes para avaliar comportamentos de crianças em atendimentos de Musicoterapia. Esses testes deram origem a duas escalas frequentemente utilizadas nos Estados Unidos da América (EUA) denominadas Escalas Nordoff Robbins (NR) de Comunicabilidade Musical e de Relação Criança Terapeuta na Experiência Musical Coativa. A partir de 1967 começou o processo de validação dessas escalas nos EUA, e em 1992 foi iniciada uma extensa revisão das mesmas baseada na prática clínica corrente no Centro Nordoff-Robbins para Musicoterapia na Universidade de Nova York. Hoje, essas ferramentas de medição são validadas e utilizadas para avaliação clínica nos atendimentos de Musicoterapia nos EUA para diversas condições médicas, entre elas, e principalmente, o autismo (NORDOFF; ROBBINS e MARCUS, 2007).

O objetivo geral dessa pesquisa foi realizar a análise psicométrica das Escalas NR de Comunicabilidade Musical e de Relação Criança Terapeuta na Experiência Musical Coativa (NORDOFF; ROBBINS e MARCUS, 2007). Os objetivos específicos foram: verificar a confiabilidade interexaminadores e a validade concorrente dessas duas escalas, na avaliação dos efeitos de intervenção da Musicoterapia Improvisacional nas crianças autistas atendidas no Hospital das Clínicas da Universidade Federal de Minas Gerais (HC-UFMG).

\section{2 - Materiais e métodos}

Foram escolhidos para análise 68 vídeos de atendimentos de crianças com diagnóstico de autismo atendidas no HC-UFMG em sessões individuais, com duração de 30 minutos cada, no método de Musicoterapia Improvisacional e modelo de Musicoterapia Musicocentrada. Foram incluídas crianças com idade entre 02 e 06 anos, e os critérios de exclusão foram: já ter recebido tratamento de Musicoterapia, ter perda auditiva profunda, hipersensibilidade auditiva e/ou ter 
ANDRÉ, Aline Moreira; BATISTA, Davi Oliveira; FREIRE, Marina Horta; SAMPAIO, Renato Tocantins; KUMMER, Arthur Melo e (2018). Análise psicométrica das Escalas Nordoff-Robbins como instrumento de avaliação no tratamento musicoterapêutico de crianças autistas em acompanhamento no Hospital das Clínicas da UFMG (HC-UFMG). Per Musi. Belo Horizonte: UFMG. p.1-12.

diagnóstico de doença neuropsiquiátrica grave. 0 ambiente de atendimento (setting musicoterapêutico) consistiu em uma sala, com armário e mesa (ambos posicionados no canto da sala, junto à parede), tatame no chão com teclado, violão (instrumentos harmônicos), flauta (instrumento melódico), voz humana e diversos instrumentos de percussão, de tamanhos e timbres bastante variados.

Quanto ao atendimento musicoterapêutico, segue uma descrição resumida: anteriormente ao atendimento, foi realizada entrevista com a família que consistiu em conversa sobre o motivo da procura do atendimento, anamnese e uma ficha musicoterapêutica, além da aplicação de dois testes específicos para avaliação de indivíduos com TEA: CARS (Childhood Autism Rating Scale) (PEREIRA, RIESGO e WAGNER, 2008) e ATEC (Autism Treatment Evaluation Checklist) (MAGIATI et al, 2011). As técnicas utilizadas no atendimento foram, principalmente, a improvisação livre e a improvisação orientada. 0 processo terapêutico se deu basicamente na detecção dos Fragmentos de Temas Clínicos e construção dos Temas clínicos (BRANDALISE, 2001; FREIRE, MOREIRA e KUMMER, 2015).

Os pacientes dos vídeos estavam divididos aleatoriamente em grupo intervenção (que havia recebido 15 sessões semanais de Musicoterapia) e grupo controle (que havia recebido apenas as sessões avaliativas inicial e final com 15 semanas de intervalo entre cada uma). Foram assistidos dois vídeos de cada paciente, com o intervalo de 15 semanas entre eles, correspondendo sempre ao primeiro e ao último atendimento do paciente. As sessões foram todas filmadas integralmente. 0 projeto foi aprovado pelo Comitê de Ética em Pesquisa da UFMG (CAAE: 03655112.3.0000.5149).

Durante a análise dos 68 vídeos foi ocultado aos examinadores: os nomes dos pacientes, o grupo que pertencia (intervenção ou controle) e a sessão correspondente (inicial ou final). Os examinadores não compartilharam resultados ou informações durante a análise.

Os examinadores aplicaram aos pacientes dos 68 vídeos as Escalas Nordoff-Robbins de Comunicabilidade Musical e na Escala de Relação Criança Terapeuta na Experiência Musical Coativa. A Escala de Comunicabilidade Musical possui três domínios: corporal, vocal e instrumental; e a Escala de Relação Criança Terapeuta na Experiência Musical Coativa possui dois domínios: Níveis de Participação e Qualidade de Resistividade. Cada domínio é dividido em 7 graus. Logo, para cada habilidade, a pontuação pode variar de 1 a 7 (NORDOFF, ROBBINS e MARCUS, 2007). Caso fosse considerado que o indivíduo se encontrava no momento de transição entre os graus da escala, pôde ser pontuado 1,5, por exemplo. Foram aplicados também os Perfis de Avaliação Improvisacional (IAPs) de Autonomia e Integração, tendo sido estabelecido que o indivíduo receberia uma nota de -2 a 2, em que a nota 0 representa o melhor perfil, em ambos os domínios (BRUSCIA, 1987).

Posteriormente, foi realizado o teste de Normalidade entre os resultados apresentados pelos examinadores para as Escalas NR e para os IAPs utilizados. A partir disso foi realizado o teste de Correlação de Spearman para verificar a confiabilidade interexaminadores.

Em outro momento, foi revelado aos examinadores a que grupo cada um dos 68 vídeos pertencia: controle ou intervenção. Também foram revelados quais vídeos eram de sessão inicial e quais eram de sessão final. No total, foram observados 19 pacientes no grupo controle 
ANDRÉ, Aline Moreira; BATISTA, Davi Oliveira; FREIRE, Marina Horta; SAMPAIO, Renato Tocantins; KUMMER, Arthur Melo e (2018). Análise psicométrica das Escalas Nordoff-Robbins como instrumento de avaliação no tratamento musicoterapêutico de crianças autistas em acompanhamento no Hospital das Clínicas da UFMG (HC-UFMG). Per Musi. Belo Horizonte: UFMG. p.1-12.

(15 meninos e 4 meninas) e 21 pacientes no grupo intervenção (18 meninos e 3 meninas). Alguns pacientes haviam pertencido ao grupo controle e posteriormente ao grupo intervenção. Foi realizado o teste de Wilcoxon (DANCEY e REIDY, 2013) para verificar os efeitos da intervenção a partir dos IAPs e das Escalas NR utilizadas na pesquisa.

Foi realizada a Correlação de Spearman para avaliar a validade concorrente das Escalas NR com IAPs, CARS e ATEC. Ressalta-se que para os testes CARS e ATEC foram utilizadas as aplicações realizadas durante a entrevista inicial com a família de cada criança.

Todas análises estatísticas foram realizadas utilizando o software SPSS 20.0. O valor de $p$ bilateral menor que 0,05 foi adotado como nível estatístico significativo para todos os testes.

\section{2 - Resultados}

Na correlação de Spearman, a partir da pontuação interexaminadores, verificou-se resultado significativo em todos os testes $(\mathrm{p}<0,001)$. Na Escala de Relação Criança Terapeuta na Experiência Musical Coativa, as correlações foram fortes em ambos domínios: Níveis de Participação $(\rho=0,791)$ e Qualidade de Resistividade $(\rho=0,756)$; e no total $(\rho=0,858)$. Na Escala de Comunicabilidade Musical, correlações fortes também foram encontradas em todos os domínios: Instrumental $(\rho=0,735)$, Vocal $(\rho=0,809)$ e Corporal $(\rho=0,820)$. No total referente à Comunicabilidade Musical, a correlação foi média $(\rho=0,695)$. Portanto, as Escalas NR apresentam uma boa confiabilidade interexaminadores.

Em relação aos atendimentos realizados no grupo intervenção, observou-se melhora significativa dos participantes segundo as Escalas NR, nos subníveis Comunicabilidade Musical Vocal $(p=0,001)$, Comunicabilidade Musical Movimento Corporal $(p=0,006)$ e no total da Comunicabilidade Musical $(\mathrm{p}=0,003)$. Encontrou-se, ainda, melhora significativa no teste IAPs referente à Autonomia $(\mathrm{p}=0,005)$ e no total $(\mathrm{p}=0,011)$.

No grupo controle, não foi observada melhora significativa em nenhum aspecto referente à análise das Escalas NR. Verificou-se apenas melhora significativa no teste IAPs, no subnível Autonomia $(\mathrm{p}=0,027)$.

As diferenças do primeiro e do último atendimento no grupo intervenção em relação à Comunicabilidade Musical Vocal e Comunicabilidade Musical Total estão apresentadas nas Figuras 1 e 2, a seguir. Verifica-se na Figura 1 que, nas sessões iniciais, a maioria dos pacientes apresentavam uma comunicabilidade musical vocal nos níveis mais baixos da escala e, nas sessões finais, houve aumento da performance destes pacientes. 
ANDRÉ, Aline Moreira; BATISTA, Davi Oliveira; FREIRE, Marina Horta; SAMPAIO, Renato Tocantins; KUMMER, Arthur Melo e (2018). Análise psicométrica das Escalas Nordoff-Robbins como instrumento de avaliação no tratamento musicoterapêutico de crianças autistas em acompanhamento no Hospital das Clínicas da UFMG (HC-UFMG). Per Musi. Belo Horizonte: UFMG. p.1-12.

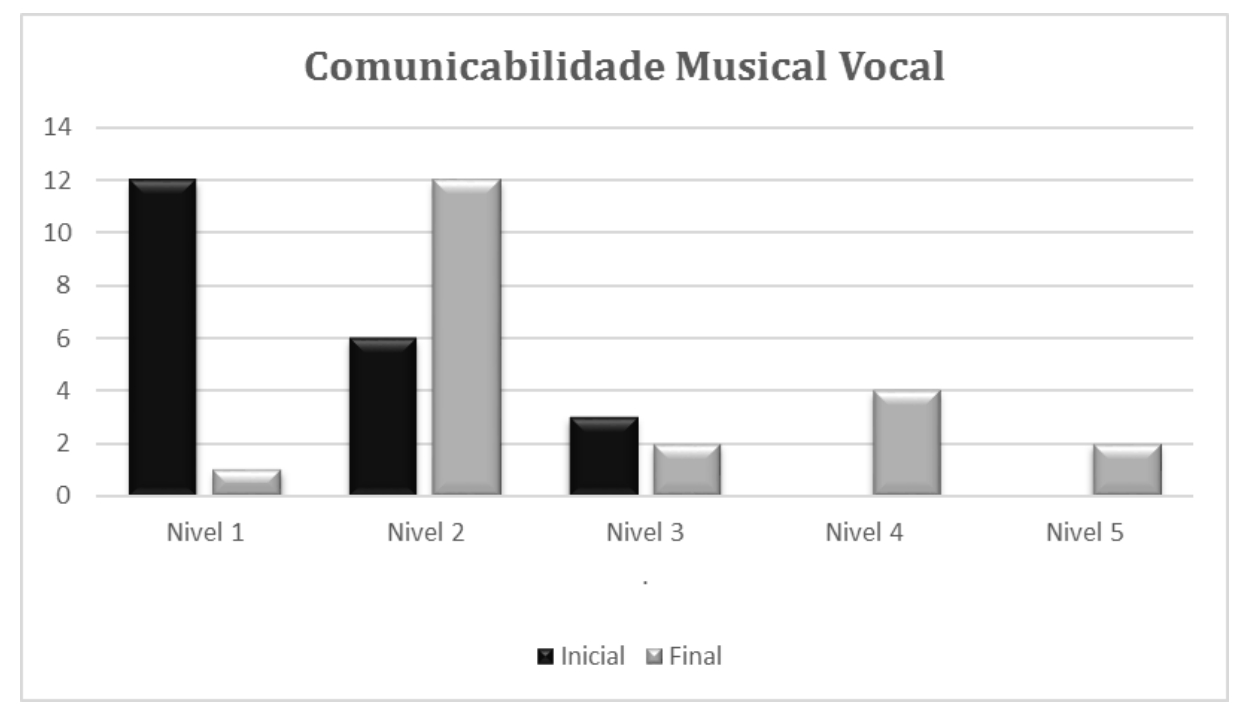

Figura 1 - Diferença de pontuação para a Escala de Comunicabilidade Musical - Dimensão Vocal, dentre as sessões iniciais e finais. No eixo vertical, encontram-se a quantidade de pacientes e no eixo horizontal, os níveis da escala.

Na Figura 2, é possível observar que nas sessões iniciais, a maioria dos pacientes apresentavam uma comunicabilidade musical nos níveis mais baixos da escala e, nas sessões finais, houve aumento geral na comunicação musical destes pacientes.

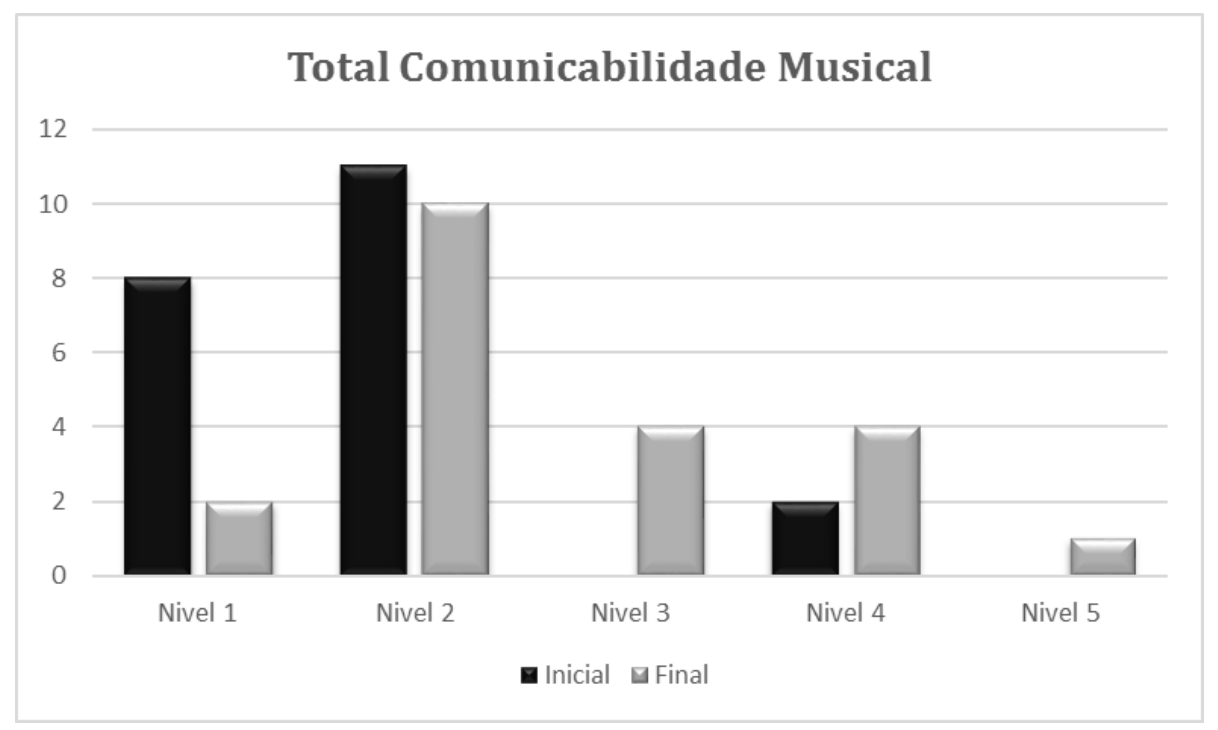

Figura 2 - Diferença de pontuação para a Escala de Comunicabilidade Musical (total), dentre as sessões iniciais e finais. No eixo vertical, encontram-se a quantidade de pacientes e, no eixo horizontal, os níveis da escala.

Como se pode observar nas Figura 1 e 2, após a intervenção da Musicoterapia, o nível de comunicação melhorou significativamente. Inicialmente, grande parte das crianças não apresentava nenhum tipo de comunicação vocal ou apresentava uma comunicação fragmentada. Após a intervenção, o nível de comunicação aumentou e o número que permaneceu sem comunicação foi mínimo. Algumas crianças conseguiram chegar a um nível de sustentação comunicacional promissor. 
Durante os atendimentos, as crianças também foram avaliadas nos testes CARS e ATEC. Correlacionamos os totais desses testes com os totais das Escalas de Relação Cliente Terapeuta e de Comunicabilidade Musical, visando avaliar a validade concorrente das escalas. Conforme apresentado na Figura 3, quatro correlações se mostraram fortes, enquanto cinco delas foram moderadas. As correlações fortes foram: CARS e ATEC, Relação Cliente Terapeuta e Comunicabilidade Musical, Relação Cliente Terapeuta e IAPs, Comunicabilidade Musical e IAPs. As correlações moderadas foram: CARS e Relação Cliente Terapeuta, CARS e Comunicabilidade Musical, CARS e IAPs, ATEC e Relação Cliente Terapeuta, ATEC e IAPs.

\begin{tabular}{|c|c|c|c|c|c|c|c|}
\hline \multicolumn{8}{|c|}{ Correlação de Sperman } \\
\hline & & & ATEC & CARS & $\begin{array}{c}\text { Relação } \\
\text { Cliente } \\
\text { Terapeuta } \\
\end{array}$ & $\begin{array}{l}\text { Comunica- } \\
\text { bilidade } \\
\text { Musical }\end{array}$ & IAPs \\
\hline \multirow[t]{5}{*}{$\begin{array}{l}\text { Spearman's } \\
\text { rho }\end{array}$} & ATEC & $\begin{array}{l}\text { Correlation Coefficient } \\
\text { Sig. (2-tailed) } \\
\mathrm{N}\end{array}$ & $\begin{array}{c}1,000 \\
40\end{array}$ & $\begin{array}{c}, 833 * * \\
, 000 \\
40\end{array}$ & $\begin{array}{c}-, \mathbf{5 3 0 * *} \\
\mathbf{0 0 0 0} \\
\mathbf{4 0}\end{array}$ & $\begin{array}{c}-, 385^{*} \\
, 014 \\
40\end{array}$ & $\begin{array}{c}, 473^{* *} \\
, 002 \\
40\end{array}$ \\
\hline & CARS & $\begin{array}{l}\text { Correlation Coefficient } \\
\text { Sig. (2-tailed) } \\
\mathrm{N} \\
\end{array}$ & $\begin{array}{c}, 833 * * \\
, 000 \\
40\end{array}$ & $\begin{array}{c}1,000 \\
40 \\
\end{array}$ & $\begin{array}{c}-, 679 * * \\
, 000 \\
40 \\
\end{array}$ & $\begin{array}{c}-, 482^{* *} \\
, 002 \\
40 \\
\end{array}$ & $\begin{array}{c}560 * * \\
, 000 \\
40 \\
\end{array}$ \\
\hline & $\begin{array}{l}\text { Relação } \\
\text { Cliente } \\
\text { Terapeuta }\end{array}$ & $\begin{array}{l}\text { Correlation Coefficient } \\
\text { Sig. (2-tailed) } \\
\mathrm{N}\end{array}$ & $\begin{array}{c}-, 530 * * \\
, 000 \\
40\end{array}$ & $\begin{array}{c}-, 679 * * \\
, 000 \\
40\end{array}$ & $\begin{array}{c}1,000 \\
40\end{array}$ & $\begin{array}{c}, 824 * * \\
, 000 \\
40\end{array}$ & $\begin{array}{c}-, 903 * * \\
, 000 \\
40\end{array}$ \\
\hline & $\begin{array}{l}\text { Comunicabi- } \\
\text { lidade } \\
\text { Musical } \\
\end{array}$ & $\begin{array}{l}\text { Correlation Coefficient } \\
\text { Sig. (2-tailed) } \\
\mathrm{N}\end{array}$ & $\begin{array}{c}-, 385^{*} \\
, 014 \\
40\end{array}$ & $\begin{array}{c}-, 482 * * \\
, 002 \\
40\end{array}$ & $\begin{array}{c}824 * * \\
, 000 \\
40\end{array}$ & $\begin{array}{c}1,000 \\
40 \\
\end{array}$ & $\begin{array}{c}-8,23^{* *} \\
, 000 \\
40 \\
\end{array}$ \\
\hline & IAPs & $\begin{array}{l}\text { Correlation Coefficient } \\
\text { Sig. (2-tailed) } \\
\mathrm{N}\end{array}$ & $\begin{array}{c}, 473^{* *} \\
, 002 \\
40\end{array}$ & $\begin{array}{c}, 560 * * \\
, 000 \\
40\end{array}$ & $\begin{array}{c}-, 903^{* *} \\
, 000 \\
40\end{array}$ & $\begin{array}{c}-8,23^{* *} \\
, 000 \\
40\end{array}$ & $\begin{array}{c}1,000 \\
40 \\
\end{array}$ \\
\hline
\end{tabular}

Figura 3 - Correlação entre os resultados obtidos nas Escalas de Relação Criança Terapeuta na Experiência Musical Coativa, Comunicabilidade Musical, ATEC, CARS e IAPs.

\section{2 - Discussão}

Tratando-se de uma área transdisciplinar, a Musicoterapia necessita de renovações constantes e diálogos com as diversas áreas com as quais se relaciona (CHAGAS e PEDRO, 2008). No decorrer dos anos observou-se que a atividade clínica e a pesquisa em Musicoterapia vêm apresentando uma necessidade de maior objetividade a fim de permitir um maior diálogo com as áreas afins.

No Brasil, a maior parte dos estudos publicados sobre Musicoterapia e autismo se refere a estudos de caso e relatos de experiência (GATTINO, 2012). As Escalas NR podem contribuir para uma avaliação mais objetiva. Existem pesquisas que realizaram a análise psicométrica de testes utilizados hoje com grande frequência, como o CARS (PEREIRA; RIESGO; WAGNER, 2008) e o ATEC (FREIRE, ANDRÉ e KUMMER, 2018 e MAGIATI et al., 2011). Essas pesquisas contribuíram para um avanço nas ferramentas de avaliação no diagnóstico clínico de autistas.

Essa pesquisa representa mais um ganho para a Musicoterapia em relação aos processos de avaliação no Brasil, sobretudo na análise dos atendimentos com crianças autistas, onde a observação e descrição é basicamente clínica. A análise dos vídeos sem ter o conhecimento de a que grupo a criança pertencia, controle ou intervenção, e a forma com a qual foi conduzida 
ANDRÉ, Aline Moreira; BATISTA, Davi Oliveira; FREIRE, Marina Horta; SAMPAIO, Renato Tocantins; KUMMER, Arthur Melo e (2018). Análise psicométrica das Escalas Nordoff-Robbins como instrumento de avaliação no tratamento musicoterapêutico de crianças autistas em acompanhamento no Hospital das Clínicas da UFMG (HC-UFMG). Per Musi. Belo Horizonte: UFMG. p.1-12.

cada sessão no desenvolvimento das habilidades avaliadas, contribuiu para uma avaliação mais objetiva.

Fatores pessoais ou familiares podem afetar o nível de participação ou comunicação em determinadas sessões. Contudo, o último atendimento provavelmente demonstrou, na maior parte, um relacionamento que foi construído ao longo dos atendimentos e uma comunicação que foi cada vez mais sustentada.

Em relação à comparação dos resultados da Escala de Relação Criança Terapeuta na Experiência Musical Coativa nas sessões de Musicoterapia inicial e final, não foram encontrados avanços significativos. Uma hipótese é que o tempo de atendimento foi curto para que os objetivos de melhora fossem alcançados com crianças dessa faixa etária e com alto grau de comprometimento. Por meio da análise desses dados percebe-se que os avanços foram mínimos e a escala pode não ter sido suficiente para representar esses pequenos avanços. Outra hipótese é que o número de crianças que tiveram melhora no Relacionamento tenha sido pequeno. Em contrapartida, acredita-se que existiu certa melhora no relacionamento que viabilizou a comunicação musical. GATTINO (2012), MØLLER, ODELL-MILLER E WIGRAM (2002) e vários outros musicoterapeutas afirmam que a improvisação é capaz de promover a interação entre os diferentes pares e permitir a auto expressão, fortalecendo as inter-relações.

No grupo intervenção, foi notada melhora na Comunicabilidade Musical Total dos pacientes, o que pode ser observado nos resultados das análises estatísticas apresentadas nas Figuras 1 e 2 (acima). Apesar disto, a melhora maior ocorreu no âmbito da comunicabilidade musical vocal e do movimento corporal, não havendo resultado significativo em relação à comunicabilidade musical instrumental. Possivelmente, a melhora vocal e de movimento corporal representam um grande desejo de comunicar-se e sem dúvida, tal melhora é bastante importante em relação ao desenvolvimento global dos indivíduos, já que representa um forte avanço na reabilitação dos mesmos. A comunicabilidade total dos indivíduos aumentou consideravelmente no grupo intervenção, havendo sustentação na comunicação para alguns dos pacientes e, para outros, o surgimento de respostas evocadas breves, porém animadoras e representantes de um prognóstico positivo. A melhora de Comunicabilidade Musical Vocal tem sido observada em outros estudos. Estudos afirmam que a música pode apresentar efeitos positivos para a comunicação e desenvolvimento da linguagem. Dentre eles, aumento de vocabulário, melhora na conversação e interação com outras pessoas (HAYOUNG, 2012).

As Escalas de Relação Criança Terapeuta na Experiência Musical Coativa e de Comunicabilidade Musical, juntamente com os IAPs, avaliam o comportamento do paciente durante o atendimento de Musicoterapia. Acredita-se que essas habilidades construídas no atendimento possam fazer parte da vida diária do paciente. Logo, uma melhora clínica em comunicação musical vocal pode representar um avanço geral de comunicabilidade vocal. Assim também, um avanço na pontuação da Comunicabilidade Musical Movimento Corporal pode representar uma melhoria no uso do corpo para a comunicação em vez de movimentos aparentemente repetitivos durante todo o tempo. Logicamente, precisamos avaliar com mais especificidade o quanto esse avanço demonstrado na clínica se apresenta de fato na vida diária. Os relatos dos pais são, até o momento, uma forma clara de verificar essas melhorias fora do atendimento. 
As Escalas Nordoff-Robbins apresentaram boas correlações entre si e com as outras escalas, como a CARS e a ATEC, o que sugere validade das mesmas. A correlação entre Comunicabilidade musical e IAPs e entre Relação Criança Terapeuta na Experiência Musical Coativa e IAPs foi significativa, indicando resultados positivos, como mostrado na Figura 3 (acima). Tais escalas estão voltadas para a sociabilidade/avaliação da comunicabilidade e da relação interpessoal dos participantes cuja correlação demonstra a eficácia da utilização e confiabilidade dos resultados, corroborando o efeito positivo que esses instrumentos de medição podem demonstrar na avaliação dos indivíduos.

Com relação às correlações de CARS e ATEC com as Escalas NR, acreditamos que o resultado não foi tão significativo porque essas análises foram feitas com a pontuação geral dos testes. Certamente se compararmos a comunicabilidade musical vocal com os subníveis de CARS e ATEC que avaliam a expressão vocal, e a relação criança-terapeuta com os domínios sociais desses testes, os resultados serão diferentes, possivelmente mais significativos.

\section{3 - Conclusão}

Dentre as várias formas de avaliar os efeitos do tratamento de Musicoterapia, verificamos que as Escalas Nordoff-Robbins de Comunicabilidade Musical e Relação Criança Terapeuta na Experiência Musical Coativa apresentaram boa confiabilidade interexaminadores e boa validade concorrente.

Com relação à intervenção, podemos verificar que a Musicoterapia Improvisacional Musicocentrada apresenta um meio de desenvolver habilidades de comunicação musical, principalmente vocal. Verificou-se que os testes ATEC e CARS se mostraram ferramentas eficientes quando utilizadas em conjunto e os IAPs apresentaram boa correlação entre si e também com as Escalas Nordoff-Robbins.

Acreditamos que a avaliação através das Escalas NR pode contribuir para definir um melhor planejamento de atendimento e avaliação de intervenção em Musicoterapia. Dessa forma, os objetivos podem auxiliar na possível conquista de habilidades e melhora da qualidade de vida através do tratamento musicoterapêutico.

Mais pesquisas são necessárias para que outros aspectos das Escalas NR sejam avaliados. Atualmente, estamos realizando estudos para verificar outros indícios de validade na utilização das Escalas NR no contexto brasileiro (ANDRÉ, 2017; ANDRÉ, GOMES e LOUREIRO, 2016, 2017). Certamente essas ferramentas contribuirão muito para que a avaliação musicoterapêutica seja cada vez mais sistematizada, objetiva e difundida.

\section{Referências}

1. AMERICAN PSYCHIATRIC ASSOCIATION. Manual diagnóstico e estatístico de transtornos mentais DSM-5. 5th ed. Porto Alegre: Artmed, 2014.

2. ANDRÉ, Aline (2017). Tradução e validação da Escala Nordoff Robbins de Comunicabilidade Musical. 108 f. Dissertação (Mestrado). Programa de Pós-Graduação em Música. Universidade Federal de Minas Gerais. Belo Horizonte. 
ANDRÉ, Aline Moreira; BATISTA, Davi Oliveira; FREIRE, Marina Horta; SAMPAIO, Renato Tocantins; KUMMER, Arthur Melo e (2018). Análise psicométrica das Escalas Nordoff-Robbins como instrumento de avaliação no tratamento musicoterapêutico de crianças autistas em acompanhamento no Hospital das Clínicas da UFMG (HC-UFMG). Per Musi. Belo Horizonte: UFMG. p.1-12.

3. ANDRÉ, A.; GOMES, C.; LOUREIRO, Cybelle (2017) Equivalência de itens, semântica e operacional da versão brasileira da Escala Nordoff Robbins de Comunicabilidade Musical. OPUS, n.2, v.23, p.197-215.

4. (2016) Escalas Nordoff Robbins: uma revisão bibliográfica. Perceptarevista de Cognição Musical, n.2, v.3, p.117-131.

5. BAIO, Jon et al (2018). Prevalence of Autism Spectrum Disorder Among Children Aged 8 Years-Autism and Developmental Disabilities Monitoring Network, 11 Sites, United States. MMWR Surveillance Summaries, n.6, v.67, p.1-23.

6. BIELENINIK, Łucja et al (2017). Effects of improvisational music therapy vs enhanced standard care on symptom severity among children with autism spectrum disorder: the TIME-A randomized clinical trial. JAMA, n.6, v.318, p.525-535.

7. BRANDALISE, André (2001). Musicoterapia músico-centrada. 1st ed. São Paulo, Brasil: Apontamentos.

8. BRUSCIA, Kenneth (1987). Improvisational models of music therapy. 1st ed. Philadelphia: Charles C Thomas Pub Ltd.

9. CHAGAS, M.; PEDRO, Rosa (2008). Musicoterapia: desafios entre a modernidade e a contemporaneidadecomo sofrem os híbridos e como se divertem. 1st ed. Rio de Janeiro: Bapera Editora.

10. DANCEY, C.; REIDY, John (2013). Estatística sem matemática para psicologia. 5th ed. Porto Alegre: Penso Editora.

11. FREIRE, Marina (2014). Efeitos da Musicoterapia Improvisacional no tratamento de crianças com Transtorno do Espectro do Autismo. 74f. Dissertação (Mestrado em Neurociências). Programa de PósGraduação em Neurociências. Universidade Federal de Minas Gerais. Belo Horizonte.

12. FREIRE, M.; ANDRÉ, A.; KUMMER, Arthur (2018). Test-retest reliability and concurrent validity of Autism Treatment Evaluation Checklist (ATEC). Jornal Brasileiro de Psiquiatria, n.1 v.67, p.63-64.

13. FREIRE, M.; MOREIRA, A.; KUMMER, Arthur (2015). Protocolo de atendimento de musicoterapia improvisacional musicocentrada para crianças com autismo. Revista Brasileira de Musicoterapia, n.18, v.1, p.104-117.

14. GATTINO, Gustavo (2012). Musicoterapia aplicada à avaliação da comunicação não verbal de crianças com transtornos do espectro autista: revisão sistemática e estudo de validação. $178 \mathrm{f}$. Tese (Doutorado). Programa de Pós-Graduação em Saúde da Criança e do Adolescente. Universidade Federal do Rio Grande do Sul. Porto Alegre.

15. MAGIATI, Iliana et al (2011). Is the Autism Treatment Evaluation Checklist a useful tool for monitoring progress in children with autism spectrum disorders? Journal of Intellectual Disability Research, n.3, v.55, p.302-312.

16. MØLLER, A.; ODELL-MILLER, H.; WIGRAM, Tony (2002). Indications in music therapy: Evidence from assessment that can identify the expectations of music therapy as a treatment for autistic spectrum disorder (ASD); meeting the challenge of evidence based practice. British Journal of Music Therapy, n.1, v.16, p.11-28.

17. NORDOFF, P.; ROBBINS, C.; MARCUS, David (2007). Creative Music Therapy: Guide to Fostering Clinical Musicianship. 2nd ed. New Hampshire: Barcelona Publishers.

18. PEREIRA, A.; RIESGO, R.; WAGNER, Mario (2008). Childhood autism: translation and validation of the Childhood Autism Rating Scale for use in Brazil. Jornal de Pediatria, n. 6, v.84, p.487-494.

19. SAMPAIO, Renato (2015). Avaliação da Sincronia Rítmica em Crianças com Transtorno do Espectro do 
ANDRÉ, Aline Moreira; BATISTA, Davi Oliveira; FREIRE, Marina Horta; SAMPAIO, Renato Tocantins; KUMMER, Arthur Melo e (2018). Análise psicométrica das Escalas Nordoff-Robbins como instrumento de avaliação no tratamento musicoterapêutico de crianças autistas em acompanhamento no Hospital das Clínicas da UFMG (HC-UFMG). Per Musi. Belo Horizonte: UFMG. p.1-12.

Autismo em Atendimento Musicoterapêutico. 138 f. Tese (Doutorado). Programa de Pós-Graduação em Neurociências. Universidade Federal de Minas Gerais. Belo Horizonte.

20. THOMPSON, G.; MCFERRAN, K.; GOLD, C (2014). Family-centred music therapy to promote social engagement in young children with severe autism spectrum disorder: a randomized controlled study. Child: care, health and development, n.6, v.40, p.840-852.

21. WIGRAM, Tony (2006). Musical creativity in children with cognitive and social impairment. In: Musical Creativity Multidisciplinary Research in Theory and Pratice. Org. by Deliège, Irène; Wiggins, Geraint. New York: Psycology Press, p.221-237.

22. WIGRAM, T.; GOLD, Christian (2006). Music therapy in the assessment and treatment of autistic spectrum disorder: clinical application and research evidence. Child: care, health and development, n.5, v.32, p.535-542.

23. WORLD FEDERATION OF MUSIC THERAPY (WFMT) (2011). President presentes... Announcing WFMT's NEW Definition of Music Therapy: o que é musicoterapia?. Montreal, Canada: WFMT. Disponível em: <http://www.wfmt.info/WFMT/President_presents..._files/President\%20 presents...5-2011.pdf>. Acesso em: 18 fev 2014

Nota sobre os autores

Aline Moreira Andre é doutoranda em Música na Universidade Federal de Minas Gerais, tem mestrado em Música (2017) e bacharelado em Música - Musicoterapia pela Universidade Federal de Minas Gerais (2014). Participou de pesquisas nos seguintes temas: protocolo de atendimento, transtorno do espectro do autismo, patologias neurológicas, musicoterapia improvisacional, musicoterapia neurológica e Escalas Nordoff Robbins.

Davi Oliveira Batista é bacharel em Música - Musicoterapia pela Universidade Federal de Minas Gerais (2014). Atualmente trabalha com performance musical e Musicoterapia.

Marina Horta Freire é musicoterapeuta, professora assistente da Escola de Música da Universidade Federal de Minas Gerais (UFMG), integrante do corpo docente do curso de Música com Habilitação em Musicoterapia dessa Universidade. Bacharel em Musicoterapia pela Universidade de Ribeirão preto (2007), mestre em Neuropsiquiatria Clínica pelo Programa de Pós-Graduação em Neurociências da UFMG (2014) e doutoranda em Educação Musical pelo Programa de Pós-graduação em Música da UFMG. Possui experiência interdisciplinar nas áreas de Saúde, Humanas, Música e Artes, com ênfase em Musicoterapia Improvisacional Musicocentrada no atendimento a pessoas com autismo ou com dificuldades emocionais.

Renato Tocantins Sampaio é professor adjunto da Universidade Federal de Minas Gerais, atuando na Graduação em Música, na Especialização em Neurociências e no Programa de Pósgraduação Stricto Sensu em Música. Tem experiência na área de Musicoterapia, Neurociências e Arte-Educação, atuando principalmente nos seguintes temas: prática clínica em musicoterapia, transtornos do desenvolvimento, educação musical e educação inclusiva. Possui graduação em Musicoterapia (1996) e em Educação Artística - Licenciatura Plena em Música (1997), mestrado em Comunicação e Semiótica (2001) e doutorado em Neurociências (2015).

Arthur Melo e Kummer é médico, realizou residência em Psiquiatria e em Psiquiatria da Infância e Adolescência e obteve Título de Especialista nessas áreas pela Associação Brasileira 
de Psiquiatria. Possui Mestrado em Clínica Médica e doutorado em Neurociências pela Universidade Federal de Minas Gerais (UFMG). Foi Professor Adjunto do Departamento de Saúde Mental da Faculdade de Medicina da UFMG entre 2010 e 2017, onde coordenou a Residência Médica em Psiquiatria da Infância e da Adolescência e os ambulatórios dessa especialidade no Hospital das Clínicas da UFMG. Foi subcoordenador e coordenador entre os anos 2014-2015 do Programa de Pós-Graduação em Neurociências da UFMG, onde era Orientador Pleno de mestrado e doutorado. Tem experiência em Neuropsiquiatria, Psiquiatria Geral e Psiquiatria da Infância e da Adolescência. Atualmente é Coordenador de Relacionamento Científico (Medical Science Liaison) da Eli Lilly and Company no Brasil. 